instillation. Cell infiltrates, expression of several inflammatory markers within bronchial lavage fluid (BALF), as well as tissue permeability were examined to evaluate the immune response and resulting lung damage.

Results Dietary Vitamin D deficient mice $(n=12)$ had elevated BALF protein concentration $(p=0.0369)$ and red blood cell $(R B C)$ extravasation $(p=0.084) 48 \mathrm{hrs}$ post IT-LPS, suggestive of increased alveolar epithelial damage. BALF levels of VEGF $(p=0.0023)$ and CXCL1/KC $(p=0.0121)$ were significantly increased $48 \mathrm{hrs}$ post-LPS, indicating an increase in the inflammatory response in deficient mice. Moreover, inflammation was prolonged with both the total number of cells recruited into the BALF ( $p=0.0479)$, and the number of apoptotic neutrophils $(p=0.034)$ significantly higher at 96hrs post LPS in Vitamin D deficient mice. Furthermore, wild type mice with normal Vitamin D levels pre-treated with cholecalciferol had reduced BALF cellular inflammation $(p=0.0093)$, with a lower BALF protein concentration $(p=0.076)$ and RBC accumulation in BALF $(p=0.0274)$ 48hrs post-LPS.

Conclusion Our data indicate that Vitamin D deficiency significantly augments both the severity and duration of murine lung injury and that exogenous Vitamin D reduces lung responses to LPS even in mice with normal Vitamin D levels. These data support the use of Vitamin D to both prevent and potentially treat established ALI.

\section{S58 SURFACTANT PHOSPHOLIPID KINETICS IN PATIENTS WITH ACUTE RESPIRATORY DISTRESS SYNDROME (ARDS)}

doi:10.1136/thoraxjnl-2012-202678.064

A Dushianthan, R Cusack, V Goss, MPW Grocott, AD Postle. Southampton NIHR Respiratory Biomedical Research Units, University Hospital Southampton, Southampton, UK

Introduction and Aims ARDS is a significant health burden. Mortality still remains high between $30-50 \%$. Surfactant is a mixture of phospholipids and proteins. Phosphatidylcholines (PC) account for $80 \%$ of total phospholipids. PC16:0/16:0 is the main PC with surface tension reducing characteristics. Surfactant abnormalities are well recognised in patients with ARDS However, replacement strategies remain unhelpful in improving mortality. Existing diagnostic definitions fail to identify a homogeneous population and this lack of phenotyping of patients according to surfactant biology may in part explain the absence of therapeutic benefit. The aims of this study are to assess surfactant PC kinetics by the incorporation of methyl- $\mathrm{D}_{9}$-choline in patients with ARDS.

Methods ARDS patients were identified according to American European Consensus Conference (AECC) criteria. Patients were infused with $3.6 \mathrm{mg} / \mathrm{kg}$ methyl- $\mathrm{D}_{9}$-choline. Small volume bronchoalveolar lavages were performed via a fibre optic bronchoscope at serial time points. Healthy volunteers were used as controls. The phospholipid fraction was extracted and analysed by triple quadrupole electrospray ionisation mass spectrometry.

Results Ten patients and nine healthy controls were recruited. The endogenous PC composition consisted primarily of PC16:0/16:0, PC16:0/18:1 and PC18:0/18:2. There was significant reduction in the relative proportion of endogenous PC16:0/16:0 in patients. Compared to healthy controls, newly synthesised deuteriated PC16:0/16:0 was much lower in patients (26\%) than controls (47\%).

Total surfactant PC $\mathrm{D}_{9}$-incorporation was linear until 48 hours $\left(0.019 \% / \mathrm{h}, \mathrm{r}^{2}=0.9734, \mathrm{P}<0.05\right)$ and reached its maximum at 48 hours $(0.93 \pm 0.15 \%)$. Steady state of incorporation was achieved between $48-96$ hours. There was $\sim 80 \%$ increase in the fractional $D_{9}$ labelling in patients at 48 hours compared to healthy controls.

Total plasma PC $\mathrm{D}_{9}$-incorporation was linear until 24 hours $\left(0.032 \% / \mathrm{h}, \quad \mathrm{r}^{2}=0.9825, \mathrm{P}<0.05\right)$ and reached its maximum $(0.755 \pm 0.056 \%)$ at 24 hours. Linear decline in enrichment was noted after 24 hours at a rate of $0.003 \%$ per hour $\left(\mathrm{r}^{2}=0.9915, \mathrm{P}<0.05\right)$. The total surfactant $\mathrm{PC} \mathrm{D}_{9}$-incorporation was much higher for patients at 24 hours and 48 hours reflecting increased synthetic rate.
Conclusions By labelling surfactant PC precursors, it is possible to study surfactant kinetics in patients with ARDS. The methodology may be utilised to phenotype patients according to alveolar surfactant kinetics prior to replacement strategies.

\section{S59 THE ROLE OF LYMPHOCYTES IN ACUTE LUNG INJURY}

doi:10.1136/thoraxjn-2012-202678.065

R Muir, M Cross, CM O'Kane, TR Craig, M Shyamsundar, DF McAuley, RJ Ingram. Queen's University Belfast, Belfast, UK

Introduction Acute lung injury (ALI) is caused by the uncontrolled activation immune cells, resulting in dysregulated inflammation which irreversibly damages the lung architecture and function. Whilst the role of myeloid cells in the pathophysiology of ALI is well established, the function of lymphoid cells is less well understood.

Hypothesis Lymphocytes may play a role inthe regulation of inflammation in acute lung injury.

Methods We have utilised both human and murine in vivo models of lipopolysaccharide(LPS) induced ALI, as well as patient samples, to characterise lung infiltrating cells by flow cytometry. Lymphocyte associated cytokines were quantified by luminex or via ex vivo stimulation andintra-cellular cytokine staining.

Results Rapid infiltration of T cells(both $\alpha \beta$ and $\alpha \beta \mathrm{T}$ cells), including regulatory $\mathrm{T}$ cells (Tregs), NKT and NKcells was observed. The cytokine Interleukin (IL)-17 is a potent recruiter of neutrophilsto sites of inflammatory sites. We observed a significant elevation in IL-17 levels in bronchoalveolar lavage fluid (BALF) in the humans postLPS inhalation; high levels of IL-17 were also seen in BALF from ALI patients. AnIL-17 KO mouse was utilised to confirm the key role for this cytokine in neutro philrecruitment to the lung during ALI. The transcription factor $\mathrm{ROR} \gamma \mathrm{t}$ induces transcription of the genes encoding IL-17. Flow cytometric analysis revealed ROR $\gamma \mathrm{t}^{+}$lung lymphocytes were predominantly $\mathrm{CD}^{4}+\mathrm{T}$ cells $(18 \%), \mathrm{CD}^{8}+\mathrm{T}$ cells (20\%)and the recently described innate lymphoid cells (40\%). Small percentages of ROR $\gamma t^{+}$natural killer (NK) cells, NK T cells, gamma delta $T$ cells were also seen. The role of Tregs in the early regulation of ALI was examined using a Treg inducibleKO murine model (FOXP3GFP DTR $)$. When Tregs were depleted prior to LPS administration there was an increased recruitment of neutrophils into the lung. Conclusion We have demonstrated that both lymphocyte released IL-17 and Tregs modulate the recruitment of neutrophils to the lung in ALI. These results reveal the importance of lymphocytes in the immunopathology of ALI. Increased understanding of the lymphocyte function could open new avenues of exploration for therapeutic strategies to regulate immune responses in ALI.

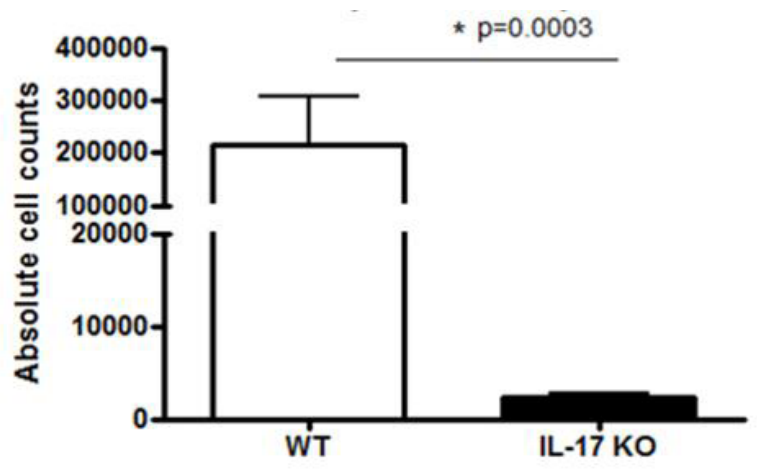

24 hrs post intra-tracheal administration of aerosolised LPS there is a significant reduction in the number of neutrophils detected within the BAL of IL-17 KO mice in comparison to wild type (WT) C57BL6 mice.

Abstract S59 Figure 1 